Argumentation et Analyse du Discours

\title{
Sallenave, Thibault. 2019. La parole impropre (Paris : Cerf)
}

\section{Ruth Amossy}

\section{(2) OpenEdition \\ 12 Journals}

\section{Electronic version}

URL: http://journals.openedition.org/aad/4372

DOI: $10.4000 /$ aad. 4372

ISSN: 1565-8961

\section{Publisher}

Université de Tel-Aviv

\section{Electronic reference}

Ruth Amossy, «Sallenave, Thibault. 2019. La parole impropre (Paris : Cerf) », Argumentation et Analyse du Discours [Online], 24 | 2020, Online since 15 April 2020, connection on 24 September 2020. URL : http://journals.openedition.org/aad/4372 ; DOI : https://doi.org/10.4000/aad.4372

This text was automatically generated on 24 September 2020.

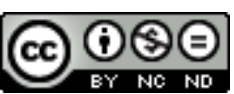

Argumentation \& analyse du discours est mis à disposition selon les termes de la licence Creative Commons Attribution - Pas d'Utilisation Commerciale - Pas de Modification 4.0 International. 


\title{
Sallenave, Thibault. 2019. La parole impropre (Paris : Cerf)
}

\author{
Ruth Amossy
}

\section{REFERENCES}

Sallenave, Thibault. 2019. La parole impropre (Paris : Cerf), 344 pages, ISBN

978-2-204-13116-2.

1 L'essai de Thibault Sallenave, La parole impropre, qui a reçu en 2019 le Prix Chaim Perelman en même temps que Rhétorique et éthique de Roselyne Koren ${ }^{1}$, traite des phénomènes de figement et de stéréotypie verbaux, ramassés dans la notion de « formularité ». Il souligne leur bivalence, et leur réversibilité intrinsèque. D’une part, ils introduisent dans notre discours le mot de l'autre, en creusant la distance qui sépare le locuteur de son dire; dans ce sens, ils sont déperdition. D'autre part, ils peuvent autoriser une rencontre réussie, un ajustement, avec l'expérience intime du sujet. L'auteur parle à ce sujet de "l'impropre » et du "propre " (on y reviendra), lesquels sont selon lui au cœur du langage et du rapport que nous entretenons avec lui. C'est de cette oscillation perpétuelle, de sa nature et de ses conditions de mise en œuvre que traite cet ouvrage touffu de 344 pages écrit par un jeune philosophe qui a choisi résolument la forme de l'essai.

Dès le début, le lecteur est pris dans un discours riche et subtil, qui déploie dans ses multiples tours et détours une réflexion nuancée. Cet art de l'écriture qui creuse les significations, éclaire des zones d'ombre, désigne des paradoxes derrière une apparente simplicité, est certes de saison pour un ouvrage qui traite des pouvoirs du langage. Plus précisément, Thibault Sallenave s'interroge sur le rapport du langage dans ses aspects conventionnels et figés (dits « formulaires »), à l'expérience vécue par le sujet dans son intimité, mais aussi dans son lien au collectif et au culturel.

3 C'est dans ce cadre que revêtent tout leur sens les qualificatifs de "propre» et «impropre » utilisés par Sallenave, un peu déroutants au premier abord et qu'il ne 
définit pas formellement. La définition de ces qualificatifs se laisse cependant saisir dans l'ensemble de l'essai, à savoir en discours. On aura remarqué que si l'expression de "parole impropre» est inusitée et attire de ce fait l'attention, elle renvoie à celle, répertoriée en langue, de mot ou expression «impropre»: "qui n'exprime pas exactement la pensée, qui n'est pas juste» (Larousse) soit adéquat, fidèle à la vérité référentielle. L'impropriété est dite dans ce même dictionnaire caractériser l'emploi incorrect d'un terme. Plus que d'incorrection au sens d'emploi erroné, il est ici question de justesse, d'adéquation de l'élément verbal à ce à quoi il renvoie. La parole impropre suppose donc au départ la possibilité d'une parole propre, comprise plus exactement dans cet ouvrage comme ajustée à l'expérience vécue du sujet (locuteur et allocutaire). Elle signale en effet pour Sallenave le passage réussi « de l'expérience en discours, de l'événement intérieur en parole, de la chose vécue en chose dite» (322-3). D'où l'utilisation récurrente des termes d'adéquation et d'inadéquation, mais aussi de justesse et d'ajustement. C'est dire que l'auteur pose implicitement au centre de sa réflexion sur la stéréotypie la possibilité - fragile, difficile, mais toujours présente et selon lui porteuse d'espoir - d'une parole susceptible de présenter «l'expression privilégiée, ajustée, réussie, d'une certaine expérience » (163).

4 Si j'insiste sur un point qui n'est pas présenté de façon autonome et systématique par Sallenave, encore moins problématisé, c'est parce qu'il est le socle sur lequel se construit tout l'édifice. Il fournit les prémisses qui permettent de développer dans sa différence une thèse qui sous d'autres aspects s'écarte assez peu de ce qui s'est écrit des années 1970 à nos jours sur la stéréotypie, sa bivalence (Amossy 1991) et le retravail des figements. En effet, l'essai pose au départ la question de savoir dans quelle mesure tous les figements dont se nourrit inévitablement la parole sont la marque d'une désappropriation, donc d'une opération par laquelle le sujet se voit privé de ce qui lui appartient en propre, et avant tout de sa subjectivité, sa " voix intérieure ". Il s'agit là d'une question qui ne cesse de resurgir sous des formes diverses depuis les écrits de Flaubert, mais aussi de Remy de Gourmont ou de Jean Paulhan, que Sallenave connaît bien, et qu'il commente avec beaucoup de finesse; mais qui resurgit aussi dans une masse importante de travaux scientifiques allant des études sur le cliché (Riffaterre, Amossy et Rosen) et sur les idées reçues (Herschberg Pierrot), aux innombrables recherches sur le stéréotype effectuées dans des domaines différents (que répertorient Amossy \& Herschberg Pierrot dans leur Stéréotypes et clichés dont la $1^{\mathrm{e}}$ édition date de 1999), et à la formule telle que théorisée dans les années 1990 par Alice Krieg-Planque. On ne peut que s'étonner de l'absence totale de cette masse critique dans un livre qui traite des questions de "formularité ", et reprend même en partie - sans pour autant s'y référer - les thèses qui s'y sont développées.

5 La particularité de l'ouvrage (signalée plus haut) est que les problèmes soulevés par les éléments figés tombés dans l'usage commun, et dès lors répétables à l'infini, y sont examinés en termes d'expérience subjective et d'expressivité. Quand Sallenave parle d'un « contact entre la parole et ce qu'il lui revient d'énoncer ", d'une adéquation entre le discours et «la chose à dire ", il entend par cette dernière non pas simplement ce dont on parle «mais une expérience complète qui engage son locuteur» (169). Ainsi, pour lui, « la formulation réussie est formulation d'une chose vécue » (167), qui engage le sujet parlant. Cette perspective permet de préciser le sens du "propre»: il " "touche" la chose, sans toutefois en capturer l'essence, dans le moment de l'expérience discursive. Il est une rencontre entre la parole et la chose à dire [...] Le propre désigne [...] la coïncidence de la parole à son objet» (169), une coïncidence 
difficile qui reste toujours fragile. Et encore : « La parole propre épouse les contours du vécu subjectif, et peut se laisser entendre comme véritablement expressive à l'égard de l'intériorité » (170).

6 L'ouvrage insiste sur le fait que sa reconnaissance n'est pas seulement affaire d'énonciation, mais aussi d'écoute. Cette dernière est particulièrement importante dans la détection de la parole formulaire et la capacité à suivre le travail qui en assure la réversibilité. En effet, Sallenave part de l'omniprésence de la formularité (traduite avant cet essai, on l'a dit, par des notions comme les idées reçues, la stéréotypie, la doxa, le préconstruit, le figement, voire le discours social) pour dépasser d'emblée la revendication romantique d'originalité dont il traite dans le premier chapitre ("Malaise dans l'authenticité »). Au-delà de l'accusation de banalité, de convention, de platitude, de mécanisation, au-delà du discours de la sottise bourgeoise rappelé à propos de Flaubert, l'auteur pose que cette " parole autre au sein de la parole propre " «n'en rejoint pas moins, par instants, la quête de sens et d'expressivité » (64). Dès lors, "même lorsque qu'une parole se donne sous la forme décevante d'une impropriété, sa faculté à rejoindre l'intimité subjective peut donner lieu à la trajectoire la plus surprenante » (12).

7 Pour prouver cette thèse, l'ouvrage accomplit un long parcours qui passe par une "Archéologie du lieu commun" (Chapitre II) où Aristote, mais aussi Cicéron ou Quintilien sont en bonne place, montrant la puissance génératrice que la rhétorique assigne aux topoï. Le Chapitre III traite de Paulhan («Vacillement de l'expression certaine »), et contient, en-dehors de considérations connues sur le cliché, un développement très intéressant sur la fonction du proverbe malgache dans l'échange verbal, où le figement "se transforme en une vérité profonde et dynamique " dans un processus d'appropriation collective. Sallenave y démontre sa thèse de la réversibilité à travers les proverbes, mais aussi les figures et les lieux communs.

8 Un chapitre focal, le chapitre IV, intitulé "L'expérience formulaire » montre que les deux réceptions possibles du formulaire sont constitutives de son fonctionnement, et qu'il contient en lui les deux versants. «Là où l'impropre devient propre - sans cependant répudier les caractéristiques de celui-ci, c'est-à-dire l'essentielle réversibilité qui lui demeure - et là où ce devenir forme le cœur de la formulation, on peut parler d"“expérience formulaire" "(173), écrit Sallenave. Cette expérience repose sur une "écoute intérieure au dire " qui peut retrouver dans l'altérité une forme d'ajustement, soit par l'attention nouvelle qui lui est prêtée soit au gré de la « trajectoire complexe de l'appropriation » (180). Celle-ci est, sans surprise, exemplifiée par les textes littéraires. L'essayiste se livre dans cet esprit à une longue et minutieuse analyse d'un extrait de Giono et de Virginia Woolf.

9 Le chapitre V, "Le devenir-fragment", explore un autre cas de figure, celui où l'impropriété - la désappropriation - sont irrémédiables mais où la parole manifeste en elle-même son incomplétude et son insuffisance. On croit y reconnaître l'obsession de la doxa et l'incapacité d'y échapper qui président à l'écriture de Gustave Flaubert ou de Roland Barthes, ou encore de Robert Pinget et de Nathalie Sarraute, pour ne citer que ceux-là. Ce n'est cependant pas vers ces références classiques que se tourne Sallenave, qui convoque plutôt Benjamin traitant de Baudelaire, ou le Austerlitz de W. G. Sebald, ou encore Reader's block, un roman de David Markson publié en 1996. Là encore, c'est la tension entre impropriété et expressivité qu'étudie l'auteur. Là aussi, on peut regretter que, pris dans sa réflexion sur le rapport à l'expérience, l'auteur ignore l'ensemble des 
textes littéraires et des réflexions critiques qui se sont déployées à partir de la prise de conscience que la doxa considérée comme désappropriation est incontournable. Une confrontation entre le point de vue de Sallenave et celui des grands écrivains et penseurs qui tenté de déjouer par l'ironie, le paradoxe, le métadiscours, etc. la parole de l'Autre tapie au cœur de leur parole propre aurait été très stimulante. Dommage que l'ouvrage en fasse l'économie.

Une dernière partie avant la conclusion est consacrée au cliché. Elle appelle à en reconnaître « un usage inventif » (207) en montrant que c'est le propre du cliché « de se donner dans l'ambivalence de la créativité et de la stérilité » (267). Nul doute qu'il s'agit là d'une position qui mérite toute notre attention - mais on ne peut ignorer qu'elle est déjà monnaie courante, et que les innombrables études du cliché, surtout dans ses usages littéraires, ont mis l'accent sur l'effet du cliché (Riffatterre 1971), ses fonctions argumentatives (Perelman et Olbrechts-Tyteca 1958), ses fonctions socio-discursives (Amossy Rosen 1982), sans compter toutes les études sur le cliché chez Flaubert, ou les ouvrages consacrés à la question en littérature et ailleurs. Sallenave, quant à lui, présente une position originale qui situe le cliché entre la catachrèse et la métaphore vive, et montre à travers quelques exemples que le cliché, s'il provoque un effacement de l'image, se situe dans une « ambivalence entre le personnel et l'impersonnel » (294) qui lui permet d'offrir une « réserve [...] d'expressivité ».

11 Un dernier chapitre (VII) qui tient lieu de conclusion, met l'accent sur le lien étroit qui relie la formularité à la culture - un développement bienvenu dans une réflexion qui met essentiellement l'accent sur l'expérience et l'expressivité individuelle. L'ouvrage souligne que dans les aspects de la formularité - qu'ils se présentent comme des figures, des proverbes, des sentences, etc. - «s'offre un certain aspect de la culture, un moyen de la parcourir et d'y circuler » (319) - sans doute toujours incertain et faillible. L'auteur distingue trois aspects de la culture formulaire: celle du vrai, qui ajuste l'expression doxique à l'expérience ; celle du répétable, qui autorise une « réactivation constante dans des contextes différents »; celle d'une distribution du propre et du commun qui permet d'insérer le second dans le premier, mais aussi d'amarrer l'individuel dans le collectif. Dans ce sens, les «expressions formulaires, dans leur variété, travaillent sur la possibilité d'une existence morale partagée » (338) - un aspect particulièrement intéressant, qui aurait mérité d'être développé dans la perspective de la relation entre rhétorique et éthique qu'explicite l'ouvrage du même nom de Roselyne Koren (2019).

Un dernier mot sur le rapport que Sallenave établit entre la parole formulaire et l'argumentation. Il note en effet qu'elle échappe au raisonnement démonstratif (322), et qu'elle ne repose pas sur «l'extension de la vérité d'une proposition à une autre proposition en vertu de leur enchaînement» (323). Et d'ajouter: la vérité de l'expression formulaire «s'effectue sans médiation, par le seul moyen de la clarté à laquelle elle peut prétendre dans une rencontre ajustée avec le vécu» (323). C'est considérer l'expression formulaire comme un fragment discursif pris en soi, en quelque sorte autonomisé et renvoyé à son extérieur (l'expérience intime, le vécu). On peut au contraire constater que l'ensemble des expressions toutes faites ont des fonctions argumentatives qu'il est possible de dégager (comme y engage la rhétorique, et en particulier la nouvelle rhétorique) en les analysant dans l'ensemble de l'énoncé ou du discours qui les mobilise. Ce sont ces éléments doxiques dans leur forme figée et 
répétable que l'argumentation dans le discours, parallèlement à d'autres travaux, étudie pour en montrer le potentiel argumentatif.

Deux remarques pour conclure : la première est une réflexion sur la forme de l'essai, et ce qu'il autorise. L'auteure de ce compte rendu est une universitaire, habituée à ce que les thèses nouvelles s'appuient sur une connaissance des travaux déjà effectués dans le domaine, souvent dans la perspective interdisciplinaire qu'appelle le sujet accoutumée aussi à ce que la nouveauté du propos jaillisse de la position assumée dans un espace déjà balisé. Sur ce point, nul doute qu'on reste sur sa faim. Cela n'empêche que le livre offre une grande satisfaction de lecture: il est riche, nourri d'une connaissance approfondie et d'une lecture fine de certains textes fondateurs, écrit dans une prose qui déclenche chez le lecteur des interrogations stimulantes et le conduit à bon port à travers un parcours cohérent et bien balisé.

Le deuxième point est qu'on trouve dans ce livre une réflexion fondée sur un idéal du langage comme adéquation à l'expérience intérieure et comme expressivité - une approche opposée à celles qui voient dans le déjà-dit la marque du dialogisme au fondement du discours. Cette position s'écarte aussi de l'approche globale qui voit dans la signification dont on investit le réel une construction verbale produite dans l'énonciation et l'interlocution. Les notions même de propre et d'impropre témoignent de ce choix. L'ouvrage va donc bravement à contre-courant des approches contemporaines qui se penchent sur le préconstruit et ses équivalents. Il aurait été bon de problématiser et discuter ce point pour souligner l'originalité de la position adoptée, en se situant dans le champ de la réflexion rhétorique, linguistique et philosophique. En même temps, il aurait fallu mentionner que la réflexion qui s'appuie avec tant de finesse et d'érudition sur la rhétorique n'adopte pas ses perspectives. L'argumentation rhétorique inspirée d'Aristote voit en effet dans le doxique le fondement même d'un discours qui vise à l'accord dans une quête d'efficacité, et non une question d'adéquation à l'expérience intime. Nul doute que de la rhétorique classique au texte de Sallenave, on constate un changement radical dans le rapport au langage. Cela aussi méritait d'être souligné, pour montrer comment la rhétorique était ici à la fois pleinement mobilisée, mais en quelque sorte déplacée.

\section{NOTES}

1. Voir le compte rendu par Emmanuelle Danblon dans ce numéro même. 


\section{AUTHORS}

\section{RUTH AMOSSY}

Université de Tal Aviv, ADARR 